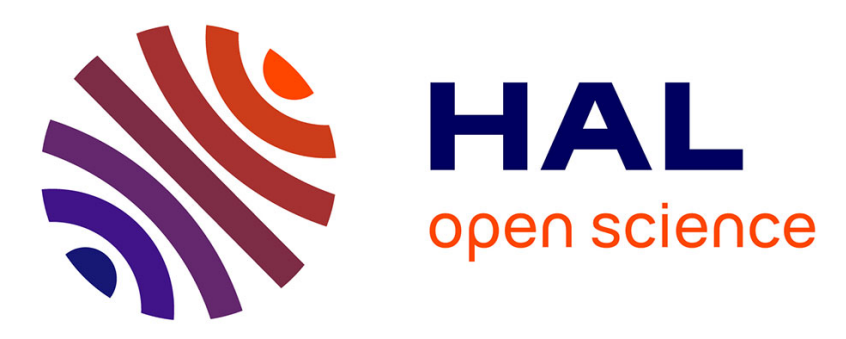

\title{
An experimental study of parameters influencing physical Human-Robot negotiation in comanipulative tracking task.
}

\author{
Lucas Roche, Anish Monachan, Ludovic Saint-Bauzel
}

\section{To cite this version:}

Lucas Roche, Anish Monachan, Ludovic Saint-Bauzel. An experimental study of parameters influencing physical Human-Robot negotiation in comanipulative tracking task.. IEEE/RSJ International Conference on Intelligent Robots and Systems, Nov 2019, Macau, China. hal-01953081v2

\section{HAL Id: hal-01953081 \\ https://hal.science/hal-01953081v2}

Submitted on 30 Dec 2019

HAL is a multi-disciplinary open access archive for the deposit and dissemination of scientific research documents, whether they are published or not. The documents may come from teaching and research institutions in France or abroad, or from public or private research centers.
L'archive ouverte pluridisciplinaire HAL, est destinée au dépôt et à la diffusion de documents scientifiques de niveau recherche, publiés ou non, émanant des établissements d'enseignement et de recherche français ou étrangers, des laboratoires publics ou privés. 


\title{
An experimental study of parameters influencing physical Human-Robot negotiation in comanipulative tracking task.
}

\author{
Lucas Roche*, Anish Monachan* and Ludovic Saint-Bauzel*
}

\begin{abstract}
The present paper investigates the relationship between human and virtual partners' behaviors during a comanipulative task requiring negotiation. More precisely, the study is focused on the influence of the Virtual Partner's (which was designed in a previous study) propensity to take the lead during conflicts over its human partner behavior. The experimental design includes both Human-Human and Human-Robot trials. The Human-Human trials serve as a baseline condition and help assessing the importance of the partner's nature (human vs robot) knowledge in the subjects behavior. The Human-Robot trials are used to observe the effect of different tuning of the Virtual Partner on the dominance relationship within Human-Robot dyads. The results of the study show that Human behavior is consistent across HumanHuman trials, and is not influenced by the knowledge of their partner's nature. Moreover, a simple tuning of the negotiation thresholds of the Virtual Partner allows to significantly modify the dominance of the human partner, although this modification has no lasting effects.
\end{abstract}

\section{INTRODUCTION}

Since the early concept of cobots [1], a significant progress in control, conception and safety has brought natural physical human-robot interaction closer to reality. Robotic devices can now smoothly interact with their environment, and react to some amount of unknown parameters. Robots are increasingly used to work alongside humans and to cooperate with them for numerous tasks in a wide range of applications, from industry to health-care [2] [3].

Communication is a crucial point for efficient cooperation, both with human and robotic partners. While visual feedback is generally the main tool used in the design of modern robots, for its simplicity and versatility, there are situations where visual feedback becomes impractical. In situations inducing a high cognitive load [4], such as surgery or piloting a plane, the visual channel can become saturated and lose its efficiency [5]. On the other hand, haptic feedback is scarcely used in current designs, although humans process it efficiently, both alone and in combination with other type of information [6] [7]. Haptic feedback also plays a key role in dyadic situations where people perform task together while in physical contact. Current research on physical HumanHuman Interaction (pHHI) shows that haptic communication occurs naturally between humans [8] [9] [10] [11] [12], and allows to increase the performance of dyads in comanipulative tasks [8] [13] [14] [15] [16] [17].

The ability for humans to communicate through the haptic channel has inspired multiple research studies aiming at

\footnotetext{
*Institut des Systèmes Intelligents et de Robotique, Sorbonne Université, 75005 Paris, France. Corresponding author ludovic.saint-bauzelesorbonne-universite.fr
}

reproducing these abilities in robots for a better physical Human-Robot Interaction (pHRI) [18][19][20][21][22]. Providing such a capability to robots could help robot designer to increase the acceptability and the clarity of the robots behavior. It is a key issue encountered when a robot is brought alongside the human to cooperate. In our previous work[16], a proof of concept that it is possible to have a virtual partner mimicking a human partner in a negotiation situation without hindering the performance of the dyad, nor changing the role dynamic of the subjects. A limitation of this proof of concept was that the parameters of the virtual partner were fixed to a set of values that was mainly due to the mechanical limitations of the device. A new device has then been developed in order to have a wider range of possibilities in torques and measures[23]. The aim of this paper is to explore the main parameters that could influence the dominance of the virtual partner in the dyad negotiation. Based on our previous observations, we hypothesize that the Leader/Follower behavior of humans can be modeled by an intrinsic time/force threshold for negotiation, which varies for each person. An experiment in pHRI settings is thus conducted to test the hypothesis and investigate the influence of the Virtual Partner's thresholds on its dominance, and the behavior of its human partners.

\section{MATERIALS AND METHOD}

\section{A. The SEMAPHORO Haptic Interface}

In order to study pHRI while controlling the information that the subjects can communicate, the choice is made to use coupled haptic interfaces and a virtual environment to realize the experimental task. The SEMAPHORO ${ }^{1}$ haptic interface [23] is designed for low-impedance, high precision tasks. It is designed specifically for the study of lightweight dyadic teleoperation. The teleoperation controller implemented in the interfaces allows to transmit forces and displacements from one interface to another, recreating the sensation of physical contact from a distance.

It is implementing a 4 channels teleoperation controller is presented in figure 1 , where the control $(\mathrm{Cm})$ is a PD controller ( $\mathrm{P}$ gain : 110; D gain : 1) and the gain transmission is proportional gain $(\mathrm{C} 2=1) . T_{d}$ represents the delay in the controller loop. It is equal to one loop period since the controller of both interfaces is run by the same process, reducing the communication delay to a minimum.

\footnotetext{
${ }^{1}$ System for human-robot physical manipulation evaluation (Système d'Évaluation de la MAnipulation Physique HOmme-RObot).
} 


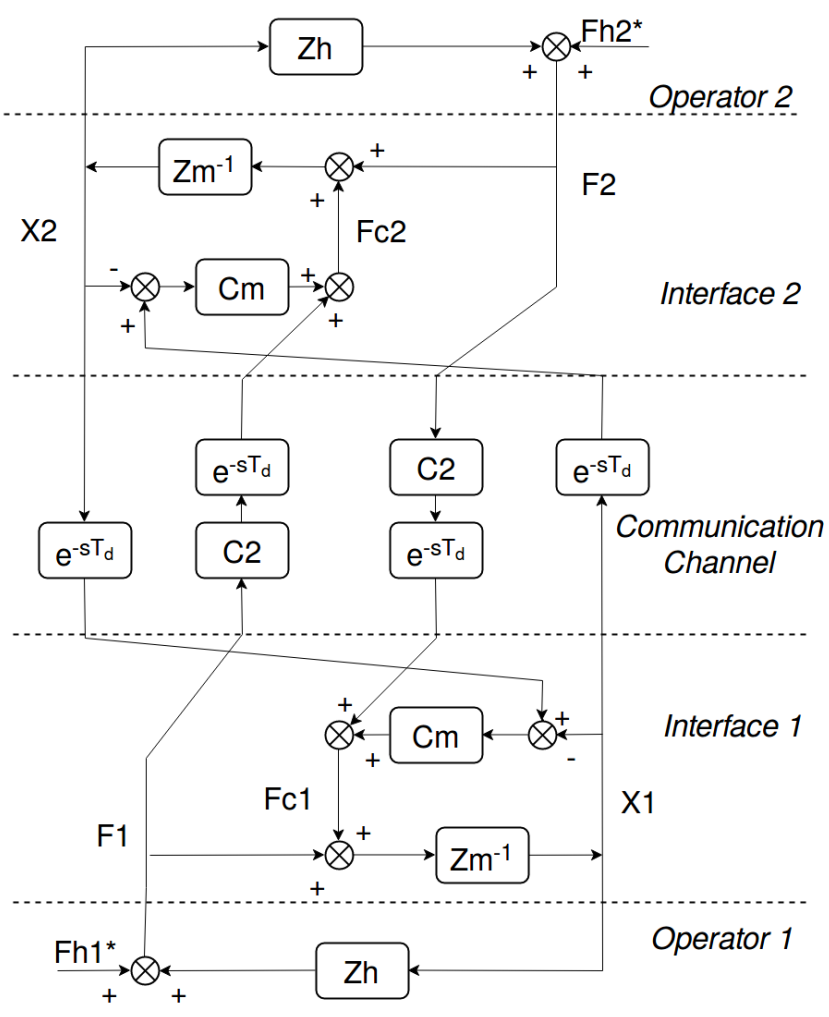

Fig. 1. Implemented 4 Channels Controller for both kinesthetic interfaces

The controller is able to provide a high precision as, it is able to measure the handle displacement with a discrete step $0.035 \mathrm{~mm}$ width. The bandwidth of the system is $10 \mathrm{~Hz}$ with a $-3 \mathrm{db}$ cut-off. The maximum force that can be transmitted at the handle is $23 \mathrm{~N}$. The total rotational inertia of the device is $J_{h}=2.96 \times 10^{-4} \mathrm{~kg} \cdot \mathrm{m}^{2}$ which represents an point-mass equivalent to $46 g$ at the tip of the handle.

\section{B. Experimental Task: Comanipulative Negotiating Task}

The experiment consists in a co-manipulative tracking task that two subjects have to complete as a dyad, with either a human or a virtual partner.

The subjects are physically separated by an opaque screen to prevent any visual clue about the actions of their partners, and wear audio headphones playing white noise to prevent any auditory clue.

A path (white line over black background) is scrolling down on their monitor, at a speed of $35 \mathrm{~mm} / \mathrm{s}$. The subjects use the haptic interfaces described previously to control the position of a massless virtual object, represented on their screen as a cursor (see Figure 2). The cursor is the same for both subjects, as they share control over a single common virtual object. The subjects are asked to keep the position of the cursor as close as possible to the scrolling path. To further incite each subject to cooperate, they are told that their goal is to maximize the common performance of the dyad. Feedback about the common performance is given by the color of the cursor, which changes based on the distance between the closest path and the cursor (see Figure 3):

- Green if $\left|X_{\text {cursor }}-X_{\text {Path }}\right|<5 \mathrm{~mm}$

- Yellow if $5 \mathrm{~mm}<\left|X_{\text {cursor }}-X_{\text {Path }}\right|<10 \mathrm{~mm}$

- Red if $\left|X_{\text {cursor }}-X_{\text {Path }}\right|>10 \mathrm{~mm}$

The path is composed of a procedurally generated succession of curves, divided in two categories:

- The "BODY" category is composed of sinusoidal-like paths of random directions but fixed duration. The purpose of these parts is to keep the subjects focused on the task between each of the studied parts.

- The "CHOICE" category is the aim of the experiment: at fixed intervals, the path splits into a fork, imposing a clear choice to be made concerning the direction that the subjects need to follow (see Figure 3). Considering that the subjects can neither see nor hear each other, the only way they can come to an agreement about the direction to choose is to use either the visual feedback from the monitor, or the haptic feedback from the handles.

While the path's structure is strictly the same for both subjects, each subject is encouraged to follow a highlighted trajectory. During the CHOICE parts, subjects receive some information about which side they have to choose [8]; this information can differ, creating situations of agreement or conflict, distributed in three cases. This is done by highlighting one of the two paths of the fork (see Figure 3):

- SAME: Both subjects have the same information, no conflict occurring.

- OPPO: Opposite information given to each subject, inducing a conflicting situation.

- ONE: Only one subject has the information. This condition forces the subjects to be ready to take initiative in case they are the only one having information about the path to choose. It is designed to discourage subjects from keeping a passive strategy all along the trials.

The subjects are informed about these choices and the different decision types beforehand.

The trials last 115 seconds, and include 2 SAME choices (both directions), 4 ONE choices (all possibilities), and 10 OPPO choices (equally distributed in the two possible possibilities) for a total of 16 choices.

\section{Virtual Partner}

An algorithm is designed to define the behavior of the virtual partner during the comanipulative task. The algorithm design is based on observations about the human behavior from previous experiments in pHHI for the experimental task. The virtual partner behaves as if it controlled a simulated handle to realize the task, creating displacements and forces perceived by its human partner during the task. The algorithm has access to information about the target trajectory, the position of its simulated handle, the position of the cursor on the monitor, the effort transmitted through its handle, and the position of its partner's handle through the position of the cursor and its own handle. The basic 


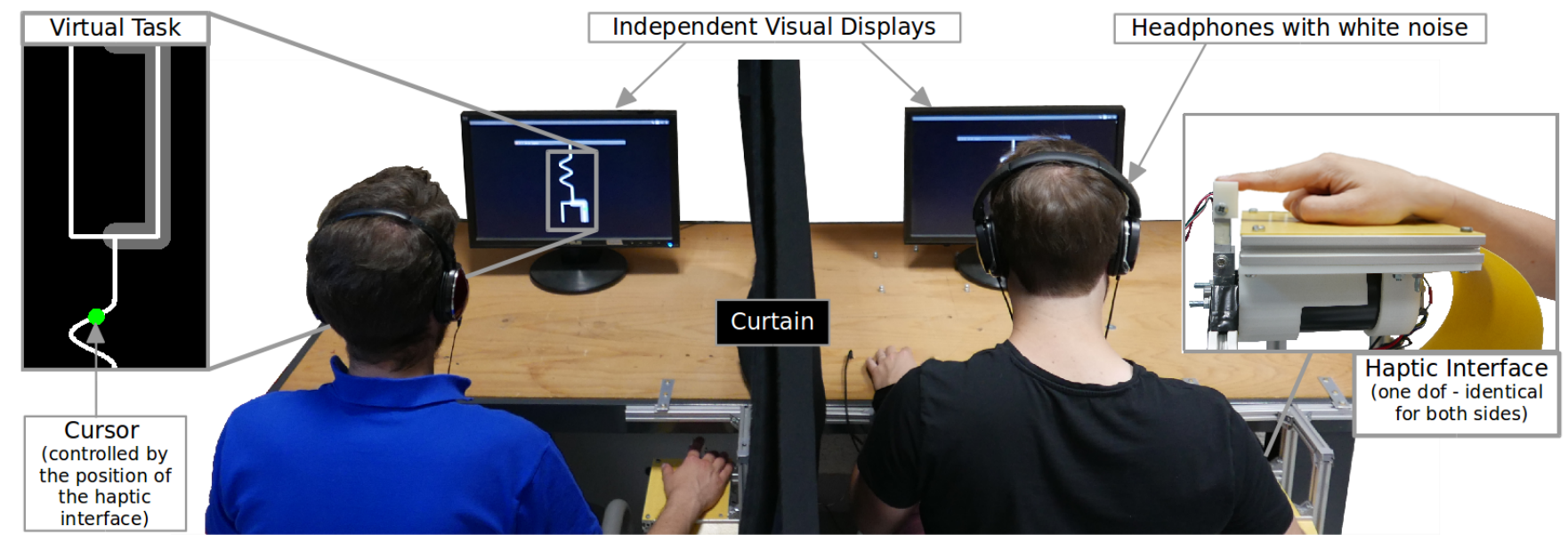

Fig. 2. Description of the experimental setup: Each participant uses a one dof haptic interface to share the control over a virtual object. Visual feedback about the position of the object is given on their respective monitors as a cursor.

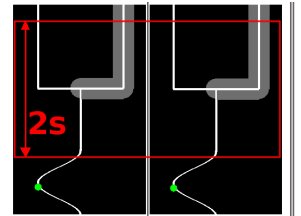

SAME

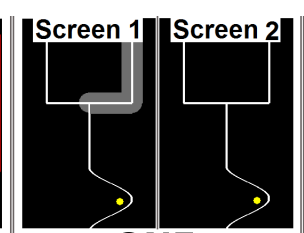

ONE

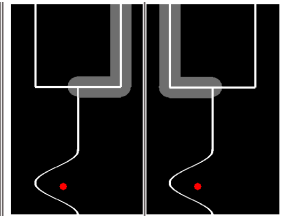

OPPO
Fig. 3. Illustration of the different decision types: SAME, ONE and OPPO. The data about the choices is recorded from a $2 \mathrm{~s}$ timezone around the path's fork (in red on the leftward figure). Visual feedback about the dyad's performance is given through the color of the cursor (from left to right : green, yellow, red).

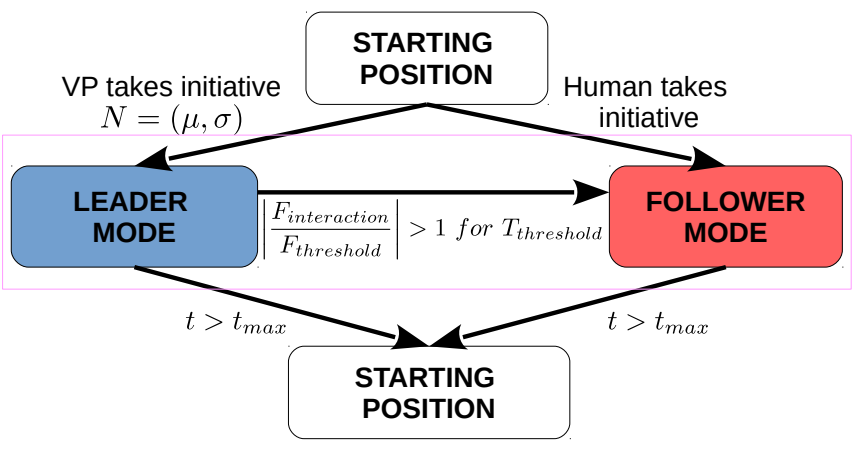

Fig. 4. Schematic functioning of the algorithm. The algorithm is designed to let the human lead the movement as a default. In the absence of human initiative, the virtual partner engage the movement toward its own target.

behavior of the Virtual Partner is explained hereafter, and more in-depth explanations can be found in [16].

In the BODY parts, the algorithm follows the path. When confronted to a CHOICE, the algorithm generates a minimum-jerk trajectory [24] from its current position to the target position, based on the choice it has to make. The minimum jerk is following this equation :

$$
X(t)=X_{o}+\left(X_{f}-X_{o}\right) *\left(10 \tau^{3}-15 \tau^{4}+6 \tau^{5}\right)
$$

where $X_{o}$ is the origin position, $X_{f}$ the final position and $\tau=\left(t-t_{\text {start }}\right) /$ Duration. The duration of the motion is generated from a normally distributed variable $(N(\mu, \sigma))$ based on the average and standard deviation of the human behavior data. Two situations are then possible: if the human takes initiative ${ }^{2}$ before the starting time of the virtual partner, it lets the human lead, entering "Follower Mode". The virtual partner generates a new trajectory to follow. This new trajectory is based on a minimum-jerk model starting at the current position of the virtual partner and ending at the new target. If the human partner did not initiate a motion before the beginning of the virtual partner's trajectory, the virtual partner takes the initiative, entering "Leader Mode", and starts its planned motion.

Once the virtual partner has started a motion in "Leader Mode", an ability to negotiate is provided in case the human wants to contest the choice. The algorithm measures the part of the interaction force between the partners which is directed toward a change of trajectory (negative if the virtual object is currently on the right, positive if the virtual object is on the left), if this interaction force exceed a fixed force threshold $F_{\text {threshold }}$ for a duration superior to a fixed time threshold $T_{\text {threshold }}$, the virtual partner switches to "Follower Mode" and generates a new trajectory to follow the human. This change in trajectory can happen multiple times if the conditions are met. Figure 4 illustrate the statemachine algorithm.

\section{Measures}

The present experiment is focused on the changes in behavior within the dyads. The data from the experiment is analyzed from the CHOICE parts of OPPO type only, in a time window of 2 seconds centered around the path forking. In OPPO choices, the dyads members have contradictory suggested trajectories, and therefore must negotiate in order to reach a common agreement on the path to take (left or right). The Dominance measure is calculated as the percentage of choices "won" by a subject, that is the

\footnotetext{
${ }^{2}$ Taking the initiative is here defined as engaging a movement of the handle resulting in a displacement of superior to $35 \%$ of the distance between the starting position and the target.
} 
number of times the dyad ended up choosing his/her/its suggested trajectory, divided by the total number of choices analyzed. More complex ways of describing the dominance have been used [25][26] but such sophistication was not deemed necessary for our analysis.

In Human-Human dyads, the subject with the highest mean dominance across the trials is designated as the Leader, while his/her partner is referred to as the Follower. In some case, data analysis for the Leader and Follower can be done separately to search for differences in Dominance patterns.

\section{E. Statistical analysis}

Statistical analysis of the results is performed using repeated measure Analysis Of VAriances(ANOVAs) [27] when there are 3 or more values of the independent variables, and with two-tailed student t-tests [28] otherwise. The student t-tests are corrected using Bonferonni correction. Results in the next sections are given with the following form: ANOVAs (F-value, p-value), t-tests (Bonferonni corrected p-value, Cohenns d coefficient for size-effect). In both methods, $\mathrm{p}$ value represents the probability to be in the NULL Condition (the same population in our case) given a statistical model and the analysis used, $p$ is compared to the significant level of $5 \%$.

\section{EXPERIMENTAL PROTOCOL}

\section{A. Experimental Conditions}

Three experimental conditions are tested during the experiment:

- Known Human Partner (KHP) In this condition, two human subjects collaborate on the task, and know that they are paired with a human. The teleoperation controller ensure that the positions of the interfaces, and thus the cursors are identifical for both subjects at all time. This additionally permits the transfer of haptic information within the dyad.

- Hidden Human Partner (HHP) : In this condition, the subjects are told they perform the task with the Virtual Partner, but are actually paired with a human partner. Outside the subject's beliefs, the rest of the parameters are identical to the KHP condition.

- Known Virtual Partner (KVP) : In this condition, the subjects are paired with the virtual partner and are correctly told so. The condition is further divided in multiple subconditions depending on the thresholds values chosen for the virtual partner.

- Hidden Virtual Partner: This condition was tested in the previous work [16] and has shown no statistical difference with KVP. Consequently, it will not be included in this protocol.

\section{B. Negotiation Thresholds: Force and Time}

The virtual partner used in the experiments includes a possibility for negotiation in cases of conflict with the human. When the robot is leading the trajectory and the human disagrees on the direction chosen, he/she can contest this choice by applying an interaction force in the opposite
TABLE I

VALUES OF $F_{\text {threshold }}$ AND $T_{\text {threshold }}$ FOR THE DIFFERENT KVP CONDITIONS.

\begin{tabular}{|c|ccc|}
\hline F Fthreshold $_{\text {threshold }}$ & 1 & 2.5 & 6.25 \\
\hline 0.2 & KVP1 & KVP3 & KVP5 \\
0.5 & KVP2 & KVP4 & KVP6 \\
\hline
\end{tabular}

direction. If the applied interaction force is superior to a fixed $F_{\text {threshold }}$ during a fixed duration $T_{\text {threshold }}$, the virtual partner yields and concedes the lead to the human.

In the previous experiments [16][17], the values of $F_{\text {threshold }}$ and $T_{\text {threshold }}$ were chosen as the average interaction forces and duration observed in the experimental data for human dyads, it was equivalent to KVP1. These thresholds however influence the behavior of the virtual partner, and thus it is reasonable to think that they can influence the behavior of the human subjects, and the role dynamic within the dyad.

The experimental design includes multiple values of $F_{\text {threshold }}$ and $T_{\text {threshold }}$. From a preliminary evaluation, thresholds values are chosen so that the difference between them is perceptible enough during the trials. Since all combinations of these thresholds must be tested in the protocol, the maximal number of threshold values must remain low, in order to keep the experiment duration acceptable. Three values of $F_{\text {threshold }}$ are chosen, and two for $T_{\text {threshold }}$, for a total of 6 combinations of KVP conditions. The chosen threshold values and following KVP conditions are shown in Table I.

\section{Hypothesis and Experimental Design}

The first hypothesis that is tested is that for any of the thresholds considered, increasing its value will increase the virtual partner dominance compared to the human subjects.

The second hypothesis is that the behavior of the subjects in human-human condition can serve as a baseline in the experimental task. The design must thus include trials in KHP condition. Moreover, in order to verify that the KHP condition is a reliable baseline, multiple trials must be included in order to verify the stability of the subject's behavior in human-human interaction.

The third hypothesis tested is that the a priori on the partner's nature has no influence on the human behavior. This hypothesis was partly validated in a previous experiment [16], where no difference were found between trials where the subjects were paired with the virtual partner and knew it (KVP), and trials where the subjects where paired with the virtual partner while thinking they were paired with a human (HVP - Hidden Virtual Partner). The reverse situation has however not yet been tested : Is there a difference between trials where the humans are together and know it (KHP), and trials where the humans think they are with the virtual partner but are actually together (HHP)?

The fourth hypothesis tested is that the Leader and Follower subjects in human dyads (KHP/HHP) behave differently when paired with the Virtual Partner (KVP). It is 
$\mathrm{H} 2$

\begin{tabular}{|c|c|c|c|c|c|c|c|c|c|c|c|}
\hline Sequence 1 : & KHP & HHP & KVP-1 & KVP-2 & KVP-6 & KVP-3 & KVP-5 & KVP-4 & & HHP & KHP \\
\hline Sequence 2 : & KHP & HHP & KVP-2 & KVP-3 & KVP-1 & KVP-4 & KVP-6 & KVP-5 & & HHP & KHP \\
\hline Sequence 3 & KHP & HHP & KVP-3 & KVP-4 & KVP-2 & KVP-5 & KVP-1 & KVP-6 & & HHP & KHP \\
\hline Sequence 4 & KHP & HHP & KVP-4 & KVP-5 & KVP-3 & KVP-6 & KVP-2 & KVP-1 & $\times 2$ & HHP & KHP \\
\hline Sequence 5 & KHP & HHP & KVP-5 & KVP-6 & KVP-4 & KVP-1 & KVP-3 & KVP-2 & & HHP & KHP \\
\hline Sequence 6 & KHP & HHP & KVP-6 & KVP-1 & KVP-5 & KVP-2 & KVP-4 & KVP-3 & & HHP & KHP \\
\hline
\end{tabular}

Fig. 5. Experimental protocol and hypothesis tested.

expected that different profiles of subjects lead them to behave more or less dominantly when paired together, and that these differences can also be seen in their behavior while paired with identical Virtual Partners.

The fifth hypothesis is that the threshold values of the virtual partner have an influence on the human behavior on the following trial(s). For example, a subject paired with an extremely dominant partner will tend to behave in a more following fashion than his/her base behavior in the next trials, even if the partner changes. In order to test this hypothesis, the experimental design must include transitions from all the different KVP conditions to a human-human condition. For practical reasons, all subjects won't provide data for all these transitions, and the data sets will be acquired over multiple dyads, at the cost of a reduced statistical test power. The transition will be done from KVP to HHP rather than KHP in order to prevent potential influence of the a priori on the partner's nature, if the previous hypothesis happens to be false.

To summarize, five hypothesis are tested during the experiment:

- H1 : Increasing threshold values leads to increasing virtual partner dominance.

- H2 : The behavior of human in human-human dyads is consistent and time invariant.

- H3 : The a priori of the partner nature has no influence on the human behavior.

- H4 : Follower and Leader in KHP conditions behave differently in KVP condition.

- H5 : Interaction with a partner of different dominance levels has lasting effects on the human behavior.

\section{Experiment Design}

The protocol includes 6 different combinations of thresholds values in the KVP conditions. In order to counterbalance the potential carryover effects, a balanced latin square design is adapted. The balanced latin square gives six different possible sequences of the experimental conditions, meaning the subjects will be grouped in 6 different categories. We decided to acquire two trials for each condition, and thus the latin square sequence was repeated twice for each subject.

The KHP condition (baseline) is tested at the start of the experiment, and at the end, which will allow to observe potential fatigue effects, or changes in behavior during the experiment. The HHP condition is also tested twice, just after the first KHP condition, which provide data for the KHP to HHP transition, and just before the last KHP condition, which provide data for the HHP to KHP condition.

Since the second HHP trials are done at the end of the KVP tests, and that the latin square design makes each sequence end with a different condition, all the six transitions from KVP to HHP are represented in the protocol, but by different subjects. Additional precautions are taken during data analysis to counterbalance the fact that not all subjects provide data for all conditions in this test. The final experimental protocol is illustrated in Figure 5, along with the tested hypothesis.

The subjects are distributed in six categories, which follow similar protocols, except for the order of the KVP conditions. Each category is tested with five subjects, for a total of 30 subjects. The 30 subjects were distributed as 15 dyads (8 male-male, 7 male-female). All participants were righthanded and had no prior knowledge about the study, experiments or experimental set-up. Data were collected from each dyad after each trial in the experiment.

\section{Results}

\section{A. Consistency of Human-Human dyads behavior}

No statistical difference is found between the first and last KHP condition $(p=0.491, d=0.13)$. The results indicates that the human dyads behavior is consistent and time invariant, hence that the KHP condition is a reliable baseline during for experiment, consistently with hypothesis $\mathrm{H} 2$.

\section{B. KHP vs HHP}

The leader won $73 \%$ of the conflicting choices in KHP condition. The leader won $67.7 \%$ conflicting choices in the HHP condition. No significant difference is found in the dominance between KHP and HHP ( $p=0.1, d=0.4)$. This result reaffirms that prior knowledge of partner's nature has no influence in the dominance, which is consistent with the hypothesis $\mathrm{H} 3$.

\section{Influence of the thresholds values}

The dominance results for the six KVP conditions can be found in Table II. Two way additive ANOVA on the six KVP conditions shows a main effect force $((F(2,492)=$ 
TABLE II

VIRTUAL PARTNER DOMINANCE FOR THE DIFFERENT KVP CONDITIONS.

\begin{tabular}{|c|c|c|c|}
\hline$F_{T_{\text {threshold }}} F_{\text {threshold }}$ & 1 & 2.5 & 6.25 \\
\hline 0.2 & $18.3 \%$ & $33.8 \%$ & $52.3 \%$ \\
\hline 0.5 & $27.3 \%$ & $42.9 \%$ & $54.9 \%$ \\
\hline
\end{tabular}

22.8, $\left.\left.p<10^{-4}\right)\right)$ and time $((F(2,492)=3.556, p=0.04))$ thresholds variations, with no interaction effect $(F(5,492)=$ 0.42, $p=0.66$ ).

Post-hoc analysis is conducted on the effects of force and time threshold variations separately. Test data is thus aggregated for the different threshold values. The virtual partner won $25.1 \%$ of the choices with the $1 \mathrm{~N}$ force threshold, $40.7 \%$ with $2.5 \mathrm{~N}$, and $54.3 \%$ with $6.25 \mathrm{~N}$. The virtual partner won $36.9 \%$ of the choices for a time threshold of $0.2 \mathrm{~s}$, and $43.2 \%$ for $0.5 \mathrm{~s}$. Results once again show significance for the influence of force $\left(F(2,492)=22.9, p<10^{-4}\right)$ and time ( $p=0.02, d=0.34$ ) thresholds. These results also suggest a stronger influence of the force threshold compared to the time threshold.

The virtual partner dominance significantly increases with the thresholds values, which confirms the hypothesis $\mathrm{H} 1$.

\section{Differences between Leader and Follower dominance}

Subject data is separated for Leader and Follower, and tests are performed to observe the influence of the thresholds for Leader and Follower subjects. The different dominance means are exposed in Figure 6.

Statistical analysis shows a significant influence of the force thresholds for both the Leader $(F(2,296)=7.591$, $\left.p<10^{-4}\right)$ and the Follower $(F(2,296)=5.248, p=0.005)$. On the contrary, no influence of the time threshold emerges for the Leader ( $p=0.14, d=0.29$ ) or Follower ( $p=0.97$, $d=0.006$ ).

Overall, there is a significant difference in dominance between the Leader and Follower, both in KHP ( $\mathrm{p}<10^{-4}$, $\mathrm{d}$ $=0.255)$, and in KVP $\left(\mathrm{p}<10^{-4}, \mathrm{~d}=0.92\right)$. The subjects that are Leaders in KHP are significantly more dominant when interacting with the Virtual Partner. This is consistent with hypothesis $\mathrm{H} 4$.

\section{E. Lasting influence of the interaction with the VP}

For this test, subject data is aggregated according to the type of the last KVP condition realized (1 to 6, see table I), with 5 subjects in each groups. A one-way between subjects ANOVA is then realized on the results in the following HHP condition, with the type of VP tuning as independent variable.

The results show no significant difference between the 6 groups $(F(5,24)=1.016, p=0.43)$, indicating that interaction with different types of Virtual Partners do not induce changes in the behavior of the subjects on subsequent trials. This hypothesis H5 is thus invalidated.

\section{F. Discussion}

The results of the experiment show that subject behavior is consistent across trials in human-human condition (KHP), moreover, a priori on the partner nature doesn't seem to affect the subject behavior (KHP vs HHP). Consequently, it can be assumed that humans exhibit an intrinsic and subject dependent tendency to dominance in negotiation situations in pHHI. It is possible that humans naturally develop and express different levels of confidence and leading behavior, and that this behavior is consistent throughout time. This result is consistent with research on Social Relation Model [29], and could be validated by conducting a similar experiment while pairing the subjects in multiple dyads combinations. This was done for example in [25], where Groten et al. found that a majority of the variability in dominance in dyads was subject dependent. The fact that interaction with the Virtual Partner does not have lasting influence on the following HHP trials further confirms the consistency of human behavior in negotiation phase.

The experimental data also shows that in pHHI, a certain imbalance exists in the dyads, as one of the subjects is systematically more dominant than the other. Additionally, humans that tend to be Leader in pHHI condition stay more dominant in pHRI than those who tend to be followers. Our results are still showing an evolution of the dominance level with both kind of persons. The results in the KVP conditions show that changing the force and time thresholds of the Virtual Partner manipulates the subjects behavior in reaction.

\section{Discussion AND CONCLUSION}

This paper shows that the force threshold mainly and the time threshold are able to influence the decision in a comanipulative tracking task during human-robot negotiation. This is interesting for two reasons. Firstly, it demonstrates a simple way to modulate the Virtual Partner behavior in "physical Human-Robot Interaction" situation. Previous research has shown that the ability to dynamically change the role allocation is a crucial point for efficient comanipulation [30] [31], and changing threshold values in our model is a way to attain this result. Secondly, if it is possible to manipulate subject behavior through changes in force and time thresholds during negotiation with the Virtual Partner, it could be possible to model that behavior using the same thresholds.

Modeling human behavior in "physical Human-Human Interaction" negotiation through a combination of time and force thresholds during interaction would allow advances in the comprehension of human behavior. It would also allow to facilitate the integration of "physical Human-Robot Interaction" protocols by enhancing the communicative ability of "Cobots". This enhancement requires to explore different kind of message that could be implemented in a Virtual Partner. For instance, the ability to choose between multiple paths (from near to far) on only one side of the path tracking or on two sides with asymmetrical paths positions. Combining different messages can be the first stones to pave the road of a kinesthetic communication. Such communication can help the human to understand naturally the robot intention/message during a physical cooperation. 

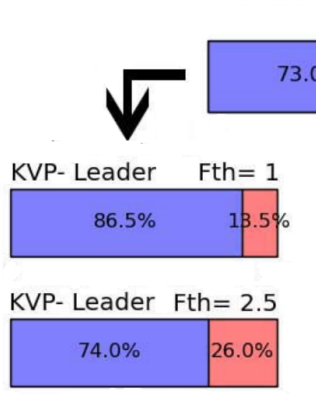

KVP- Leader Fth $=6.25$

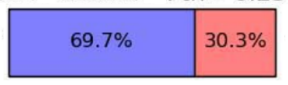

KHP
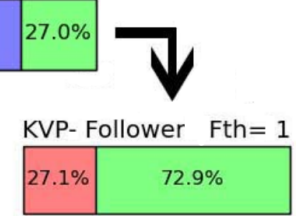

KVP- Follower Fth $=2.5$

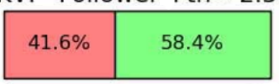

KVP- Follower Fth $=6.25$

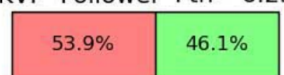

$\mathrm{KHP}$
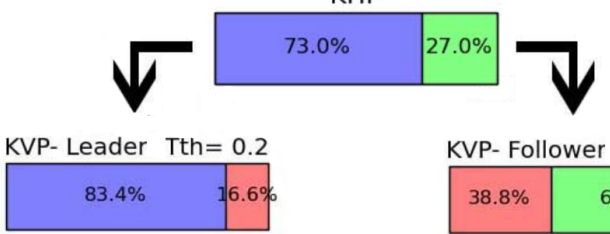

KVP- Leader Tth $=0.5$

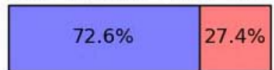

KVP- Follower Tth $=0.5$

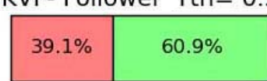

Fig. 6. Dominance results for Leader and Follower in the different threshold conditions

In order to improve our Virtual Partners, we will need to take into account the possible inter-individual variations of behavior in order to ensure the best cooperation and thus the best performances for Human-Robot comanipulation.

\section{REFERENCES}

[1] M. Peshkin and J. E. Colgate, "Cobot architecture," IEEE Tansactions on Robotics and Automation, Vol 17, No 4, 2001.

[2] A. D. Santis, B. Siliciano, A. D. Luca, and A. Bicchi, "An atlas of physical human-robot interaction," Mechanism and Machine Theory, 2007.

[3] M. A. Goodrich and A. C. Schultz, "Human-robot interaction : A survey," Foundations and Trends in Human-Computer Interaction, 2007.

[4] G. A. Miller, "The magical number seven, plus or minus two: some limits on our capacity for processing information," Psychological Review, 1956.

[5] H. A. H. C. van Veen and J. B. F. van Erp, "Tactile information presentation in the cockpit," in Haptic Human-Computer Interaction (S. Brewster and R. Murray-Smith, eds.), (Berlin, Heidelberg), pp. 174-181, Springer Berlin Heidelberg, 2001.

[6] L. A. Jones, "Kinesthetic sensing," 2000.

[7] M. O. Ernst and M. S. Banks, "Humans integrate visual and haptic information in a statistically optimal fashion," Nature, vol. 415, 2002.

[8] R. Groten, D. Feth, R. L. Klatzky, and A. Peer, "The role of haptic feedback for the integration of intentions in shared task execution," IEEE Transactions on Haptics, Vol 6, No 1, 2013.

[9] J. Moll and E.-L. Sallnas, "Communicative functions of haptic feedback," Haptic and Audio Interaction Design, 2009.

[10] A. Sawers, T. Bhattacharjee, J. L. McKay, M. E. Hackney, C. C. Kemp, and L. H. Ting, "Small forces that differ with prior motor experience can communicate movement goals during human-human physical interaction," Journal of NeuroEngineering and Rehabilitation, 2017.

[11] R. P. V. der Wel, G. Knoblitch, and N. Sebanz, "Let the force be with us: Dyads exploit haptic coupling for coordination," in Journal of Experimental Psychology: Human Perception and Performance, 2010.

[12] C. A. C. Parker and E. A. Croft, "Experimental investigation of humanrobot cooperative carrying," IEEE/RSJ International Conference on Intelligent Robots and Systems, 2011.

[13] S. Glynn, R. Fekieta, and R. A. Henning, "Use of force-feedback joysticks to promote teamwork in virtual teleoperation," in Proceedings of the Human Factors and Ergonomics Society 45th Annual Meeting, 2001.

[14] G. Ganesh, A. Tagaki, T. Yoshioka, M. Kawato, and E. Burdet, "Two is better than one: Physical interactions improve motor performance in humans," Nature, Scientific Report 4:3824, 2014.

[15] A. Takagi, F. Usai, G. Ganesh, V. Sanguineti, and E. Burdet, "Haptic communication between humans is tuned by the hard or soft mechanics of interaction," PLOS Computational Biology, vol. 14, pp. 1-17, 03 2018.
[16] L. Roche and L. Saint-Bauzel, "Implementation of haptic communication in comanipulative tasks: A statistical state machine model," in 2016 IEEE/RSJ International Conference on Intelligent Robots and Systems (IROS), pp. 2670-2675, Oct 2016.

[17] L. Roche and L. Saint-Bauzel, "High stiffness in teleoperated comanipulation: necessity or luxury ?," in IEEE International Conference on Robotics and Automation, 2018.

[18] Y. Aydin, N. Arghavani, and C. Basdogan, "A new control architecture for physical human-robot interaction based on haptic communication," Proceedings of the 2014 ACM/IEEE international conference on Human-robot interaction, 2014.

[19] P. Evrard and A. Kheddar, "Homotopy switching model for dyad haptic interaction in physical collaborative tasks," in Third Joint Eurohaptics Conference and Symposium on Haptic Interfaces for Virtual Environment and Teleoperated Systems, 2009.

[20] G. J. Maeda, G. Neumann, M. Ewerto, R. Lioutikov, O. Kroemer, and J. Peters, "Probabilistic movement primitives for coordination of multiple human-robot collaborative tasks," Autonomous Robots, pp. 593-612, 2017.

[21] A. Mortl, M. Lawitzky, A. Kucukyilmaz, M. Sezgin, C. Basdogan, and S. Hirche, "The role of roles: Physical cooperation between humans and robots," The International Journal of Robotics Research, vol. 31, no. 13, pp. 1656-1674, 2012.

[22] A. Kucukyilmaz, T. M. Sezgin, and C. Basdogan, "Intention recognition for dynamic role exchange in haptic collaboration," IEEE Transactions on Haptics, 2014.

[23] L. Roche and L. Saint-Bauzel, "The semaphoro haptic interface: a realtime low-cost open-source implementation for dyadic teleoperation," in ERTS, 2018.

[24] T. Flash and N. Hogan, "The coordination of arm movements: an experimentally confirmed mathematical model," The journal of Neuroscience, vol. 5, no. 7, pp. 1688-1703, 1985.

[25] R. Groten, J. Holldampf, A. Peer, and M. Buss, "Predictability of a human partner in a pursuit tracking task without haptic feedback," Second International Conferences on Advances in Computer-Human Interactions, 2009.

[26] N. Stefanov, A. Peer, and M. Buss, "Role determination in humanhuman interaction," in Third Joint Eurohaptics Conference and Symposium on Haptic Interfaces for Virtual Environment and Teleoperator Systems, 2009.

[27] C. S. Davis, Statistical methods for the analysis of repeated measurements. Springer Science \& Business Media, 2002.

[28] S. Senn and W. Richardson, "The first t-test," Statistics in Medicine, vol. 13, no. 8, pp. 785-803, 1994.

[29] D. A. Kenny, D. Mohr, and M. J. Levesque, "A social relations variance partitioning of dyadic behavior," Psychological Bulletin, 2001.

[30] N. Jarasse, V. Sanguinetti, and E. Burdet, "Slaves no longer : review on role assignment for human-robot joint motor action," Adaptative Behavior, 2013.

[31] D. A. Abbink, M. Mulder, and E. R. Boer, "Haptic shared control: smoothly shifting control authority?," Cognitive Tech Work, 2012. 